\section{Continuous quality improvement in practice: lessons from the RCVS Knowledge award winners}

\section{Lesley Moore, Louise Northway and Alison Thomas}

Quality improvement (QI) initiatives within practice can help towards improving patient safety, outcomes, client satisfaction and team morale. Getting started with these initiatives and keeping up the momentum within the team can be challenging, but it does not have to be.

- Louise Northway RVN, also known as Lou the Vet Nurse, will talk about her role at Wendover Heights Veterinary Centre and how she works with the team to undertake clinical audits and to set and review protocols based on evidence. Louise will talk about how and why her practice is motivated to improve continuously

- Alison Thomas MRCVS from Blue Cross will discuss her project to deliver a consistent approach to diagnosis and treatment throughout the charity by developing guidelines. Alison will talk about how Blue Cross supported her to give her the time to focus on this project, and how she secured team buy-in so that everyone in the charity uses the guidelines

- Lesley Moore RVN, will talk about how she lead the nursing team at Vets Now Macclesfield to use clinical auditing to improve the quality of recording of anaesthesia and sedation monitoring which led to zero significant events and an improvement in patient outcomes.
Delegates will hear how the award winners took simple steps, which can be replicated in other practices, to achieve measurable and improved outcomes for their practices and their patients. They will talk about some of the challenges they overcame and they will show how QI can help to build a strong team ethos.

\section{KEY LEARNING OBJECTIVES}

- To show what good quality improvement (QI) looks like

- Practical advice on how to get started with continuous QI in practice

- Real examples of the positive effect of QI on patient outcomes and team morale

\section{MULTIPLE CHOICE QUESTIONS}

1. How can you decide what to improve?

(A) Use information from your client feedback forms

(B) Identify where improvements can be made during a team discussion

(C) Repeat a topic based on your previous work experience

(D) All of the above

2. What techniques does RCVS Knowledge recommend for practices to take a structured approach for continuous QI?
(A) Benchmarking
(B) Clinical audit
(C) Protocols, checklists and guidelines
(D) All of the above in an ongoing cycle

3. What can clinical audit help improve?

(A) Nothing - clinical audit is just a way to measure what we do

(B) Patient care and outcomes

(C) B and D when the results from the audit are investigated, then used to develop improvement strategies in practice

(D) Client waiting times 